\title{
Interdisziplinäres Therapiekonzept
}

Der Mai ist gekommen - und wieder laden interessante und hochkarätig besetzte Fortbildungsveranstaltungen und Dentalausstellungen jeden Zahnarzt dazu ein, sein Wissen und auch sein operatives Repertoire zu erweitern. Fortbildung ist auch unsere Aufgabe, und daher bieten wir Ihnen in dieser Ausgabe wieder eine breite Palette an Inhalten.

Fallberichte, wie der aktuelle Beitrag „Interdisziplinäres Therapiekonzept bei unilateraler Lähmung der Kaumuskulatur“, tragen zum Leben der Zeitschrift bei und machen auch den Stellenwert der Zahnmedizin deutlich: Unser Fach ist eingebettet in zahlreiche Rahmenfächer und zudem in die „große Medizin“. Kein Patient, der sich mit einem Zahnproblem in einer zahnärztlichen Ordination einstellt, ist ansonsten ein „weißes Blatt“, jeder hat seine eigene Lebens- und Gesundheitsgeschichte. - Manche Erkrankungen machen einen interdisziplinären Therapieansatz erforderlich.

Neue Behandlungsmethoden, wie das Arbeiten mit CAD/CAM, bieten immer ausgereiftere technische Vorrichtungen an, sodass wir uns entschlossen haben, auch dieses „heiße Eisen“ einmal anzugreifen: Lesen Sie den interessanten und informativen Beitrag über Weiterentwicklungen in der CAD/CAM Technologie.

So weit ein solches System entwickelt sein kann, es ersetzt die menschliche Interaktion nicht: Im Gegenteil, der Patient orientiert sich primär an der Art, wie mit ihm umgegangen wird, und auch daran, wie die an seiner Behandlung Beteiligten miteinander arbeiten und kommunizieren. In erster Linie möchten Patienten beim Zahnarzt individuell und respektvoll behandelt werden, dann erst orientieren sie sich an der fachlichen Kompetenz und am Verlauf der Behandlung, um zu einer Einschätzung ihres Arztes zu kommen, wie Umfragen zeigen.

Wieder einmal thematisieren wir das Arzt-Patienten Gespräch: Die Qualität der Kommunikation muss passen, und zwar in alle Richtungen, interdisziplinär und im eigenen Team, vor allem aber von allen Team- Mitgliedern mit dem Patienten.

Wir wünschen Ihnen viel Vergnügen beim Lesen!

Ihr Stomatologie-Redaktionsteam 